\title{
Non-dipolarity of Heart Potentials Estimated by Magnetocardiography in Normal Subjects
}

\author{
Naomi Izumida,' MD, Yuh Asano, ${ }^{1}$ MD, \\ Junro Hosaki, ${ }^{2} \mathrm{MD}$, Yasunaga Hroshi, ${ }^{,} \mathrm{MD}$, \\ Harumizu SAKURADA, ${ }^{+}$MD, Takeshi MотомIYa, ${ }^{4} \mathrm{MD}$, \\ Seiko Kawano, ${ }^{3}$ MD, Tohru SawanoborI, ${ }^{3}$ MD \\ and Masayasu HiraokA, ${ }^{3} \mathrm{MD}$, FACC
}

\section{SUMMARY}

We studied non-dipolarity characteristics during ventricular excitation in normal adults and children by magnetocardiography (MCG) by recording magnetic field on the thorax. The source and currents of the electrical dipole from the onset up to $60 \mathrm{~ms}$ of ventricular excitation were analyzed in 16 adults and 5 children. A single equivalent current dipole (ECD) was estimated by Sarvas' formula for the sphere model at $1 \mathrm{~ms}$ intervals. The non-dipole value (NDV) was calculated from the magnetic field strength at each recording point and theoretically estimated by ECD, representing an index for the nondipolarity. At 32-34 ms from the beginning of QRS, the mean NDV was a minimum in all subjects suggesting at least a non-dipole component during this period. High NDV (over 5\%) were present in most subjects in both the early and late phase compared to this period. Thirteen of 16 adults had a high NDV in the early phase $(9.3 \pm 3.0 \%$, mean $\pm \mathrm{SD})$ and all 16 subjects had a high NDV in the late phase $(21.5 \pm 10.5 \%)$. All 5 children had high NDV in both the early $(10.5 \pm 5.4 \%)$ and late phases $(16.8 \pm 7.9 \%)$. A single ECD estimation by MCG showed a relatively low non dipolar component and MCG could be applied to the clinical evaluation of cardiac excitation in both normal and pathological conditions. (Jpn Heart J 1998; 39: 731-742)

Key words: Magnetic field, Equivalent current dipole, Non-dipolarity

$\mathrm{E}$ LECTRICAL activity of the heart can be simulated as a single moving dipole. The dipole, if exactly determined, could be used to ascertain the origin of an ectopic focus, abnormal conduction pattern in WPW syndrome, ${ }^{1-3)}$ and so on. There have only been a few reports which evaluated the reliability of

From 'Department of Pediatrics, School of Medicine, 'School of Allied Health Sciences, and, ${ }^{3}$ Department of Cardiovascular Disease, Medical Research Institute, Tokyo Medical and Dental L'niversity, Tokyo, and, ${ }^{4}$ Department of Cardiology, Tokyo Metropolitan Hiroo Hospital, Tokyo, Japan.

Address for correspondence: Naomi Izumida, MD, Deparment of Pediatrics, School of Medicine, Tokyo Medical and Dental University, 1-5-45 Yushima, Bunkyo-ku, Tokyo 113-8519, Japan.

Received for publication August 3, 1998.

Revised and accepted September 28, 1998. 
a single dipole for cardiac electrical activity. ${ }^{4-6)}$

Estimation of the source current and non dipole component in the in situ heart has been done through inspection or measurement of the electrocardiographic and vectorcardiographic wave forms. Multiple and simultaneous electrical recordings from the thoracic surface revealed a contribution of multiple generators during ventricular depolarization. ${ }^{4,5}$

Magnetic measurement is another method with which to detect the electrical activity of the heart. Electrical current flow from the generator produces a magnetic field surrounding the current source according to the corkscrew law. Recent advances in new technologies make it possible to detect in the human body a weak magnetic field that is produced by electrical activity of excitable organs such as brain and heart. ${ }^{7-10}$ The magnetic field on the thoracic surface is less distorted by different body builds and air contents surrounding the generator $^{1,2,11)}$ than the electric field. Therefore, magnetocardiography (MCG) may be a noninvasive and accurate method for analyzing cardiac electrical activity in situ.

The aim of the present study was to examine the dipolarity characteristics of the ventricular excitation by MCG.

\section{Subjects and Methods}

Subjects: Sixteen healthy adults (range: $25-55$ years old, mean 34.4 years old) and 5 children ( 6 to 12 years old) were enrolled in the study. The absence of any structural heart disease was verified in all 21 subjects by physical examination and ECG and chest X-ray findings. Informed consent was obtained from each subject or their parent or legal guardian prior to performing the MCG procedure.

MCG measurements: MCG measurement were obtained using a 37 channel biomagnetometer (model SMI-1004: jointly manufactured by Sumitomo Metal Industries Ltd., Tokyo and Biomagnetic Technologies Inc, San Diego, USA) in a magnetically shielded room (type Ak 3b: Vacuumschmelze GmbH, Hanau, Germany). A limb lead ECG was recorded simultaneously. The layout of the 37 channels in a sensor unit of the biomagnetometer is shown in Figure lA. The base of the sensor was concave and the channels were arranged to conform to its shape. The sensor unit had a diameter of $12.6 \mathrm{~cm}$ and did not cover the entire chest. Therefore, in order to measure the magnetic fields over the entire chest, the sensor unit was moved to 6 different positions on the anterior chest as shown in Figure 1A. With the subjects laying in a supine position on the bed, the sensor was positioned with its center (No. I channel) on one of the 6 points $(\mathrm{C}, \mathrm{RU}, \mathrm{RL}$, LU, LL and LM) as close as possible to the chest wall. Point $\mathrm{C}$ was located in the 5 th intercostal space at the anterior median line. Points RU, RL, LU, and LL 


\section{Sensor placements and recorded MCG data}

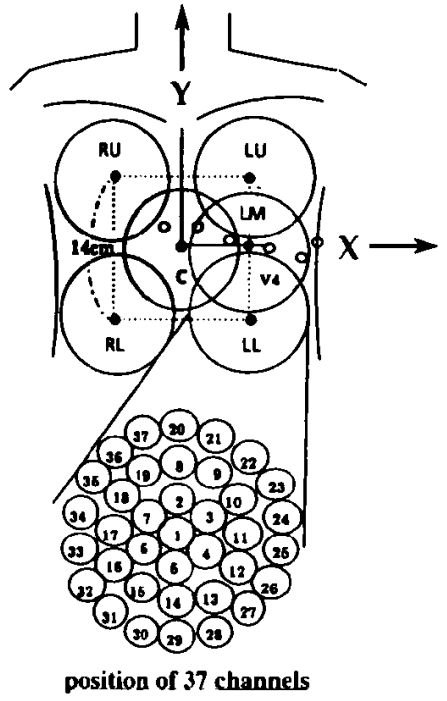

A

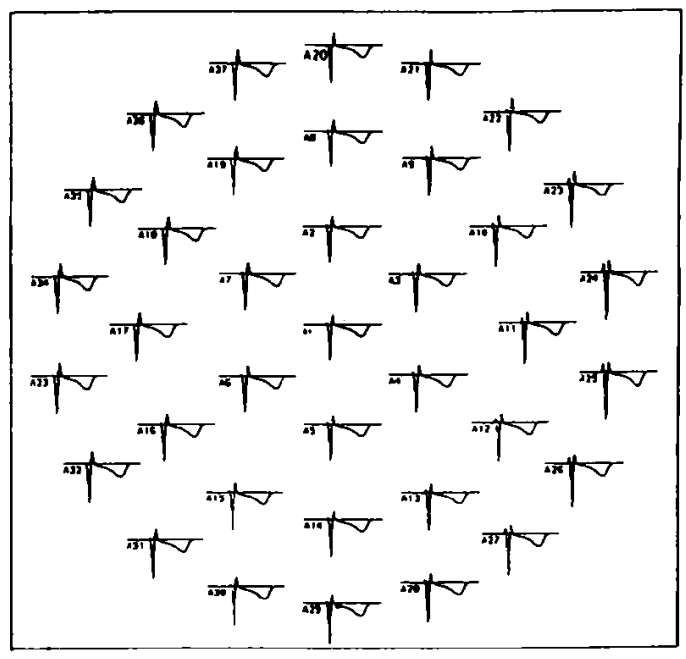

B

Figure 1. Sensor placements of the recording system and recorded MCG data. A: Placement of 37 channel gradiometer over the body. Sensor was positioned with its center (No 1 channel) just above points $\mathrm{C}, \mathrm{RL}, \mathrm{RL}, \mathrm{LU}, \mathrm{LL}$ and $\mathrm{LM}$. Point $\mathrm{C}$ was located in the 5 th intercostal space at the anterior median line. Points RU, RL, LL' and LL are the four corners of a square with $14 \mathrm{~cm}$ sides. LMI was located midway between $\mathrm{LL}$ and $\mathrm{LL}$. Open circles indicate the positions of the precordial leads of standard ECG. B: Recorded waves of MCG. The waves by MCG were very similar to those of ECG recordings.

were the 4 corners of the $14 \mathrm{~cm} \times 14 \mathrm{~cm}$ square. LM was the point midway between LU and LL and was used to obtain supplementary information on the left side of the thorax. For children, the center position was the same as the adults, however, the other positions were modified due to their smaller body size. Recordings were made at each position for 2 or 3 minutes to acquire data representing about 100 sinus beats. The sampling interval was 1 millisecond (ms).

Data processing: MCG wave data for each channel were clustered according to their wave morphologies. The wave data in each cluster were averaged and the average wave of the most frequent morphology for each channel was used in the subsequent analysis. Waves of over 100 beats were usually added, averaged and then calibrated according to time and baseline. Time calibration was performed by detecting the timing of the onset of QRS in the simultaneously recorded lead II of the ECG wave and was corrected with every MCG wave from all channels. Low-frequency noise artifacts in the MCG data was reduced by high-pass filtering $(0.1 \mathrm{~Hz})$ and the baseline was adjusted by subtraction of an 
interpolated polynomial curve with a terminal portion of the TP segment as the reference level.

Isomagnetic map display and dipole estimation (Figure 2): Measured magnetic field values were corrected their strength to perpendicular component to the sensor and an isomagnetic map of the plane at the level of the center channel of the sensor was displayed. The magnetic layer in the direction from the body toward the sensor as positive was defined and the layer in the opposite direction as negative. The distributions of the magnetic layers were displayed with the positive and negative areas of isomagnetic lines at intervals of $1 \mathrm{pT}$ during the ventricular excitation.

For the dipole estimation, the thorax was imaged as a sphere with homog-

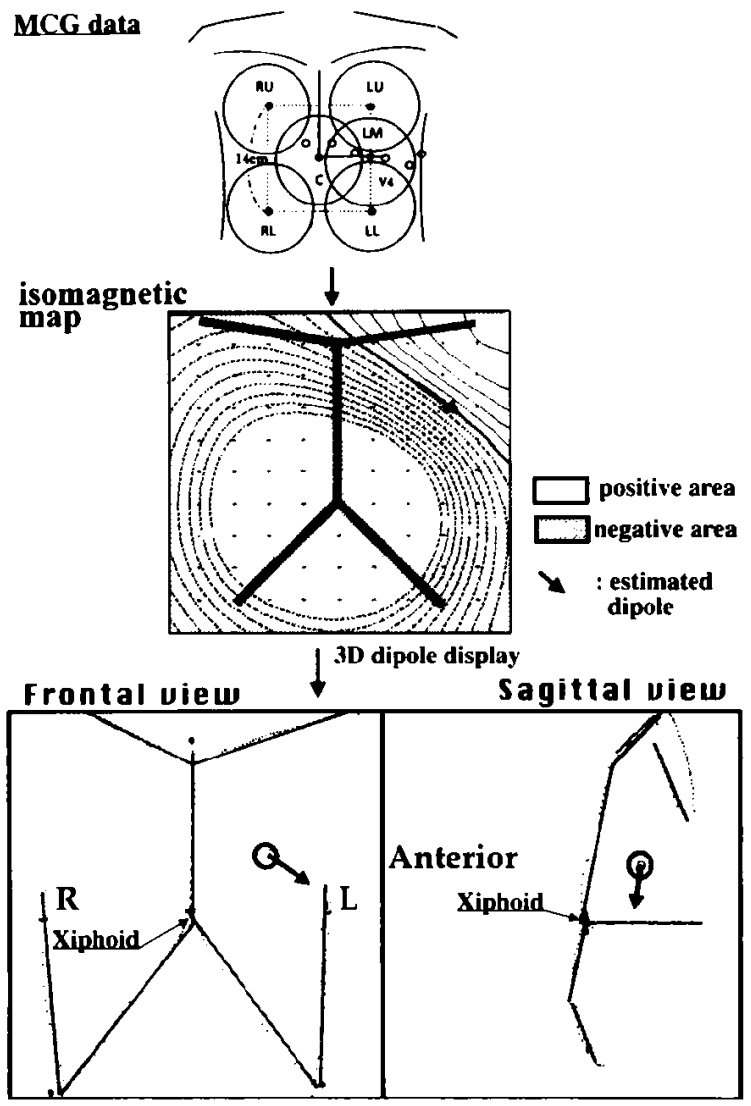

Figure 2. Schema for analyzing process of MCG data. MCG data were processed as described in the text and an isomagnetic map of the thorax was constructed at each instance of the analysis. A single electrical equivalent dipole was estimated from the distributions of the magnetic field. The estimated dipole was then displayed 3 dimensionally with its position, direction, and strength. The process was repeated during the time of analysis and the serial changes of NDV displayed. 


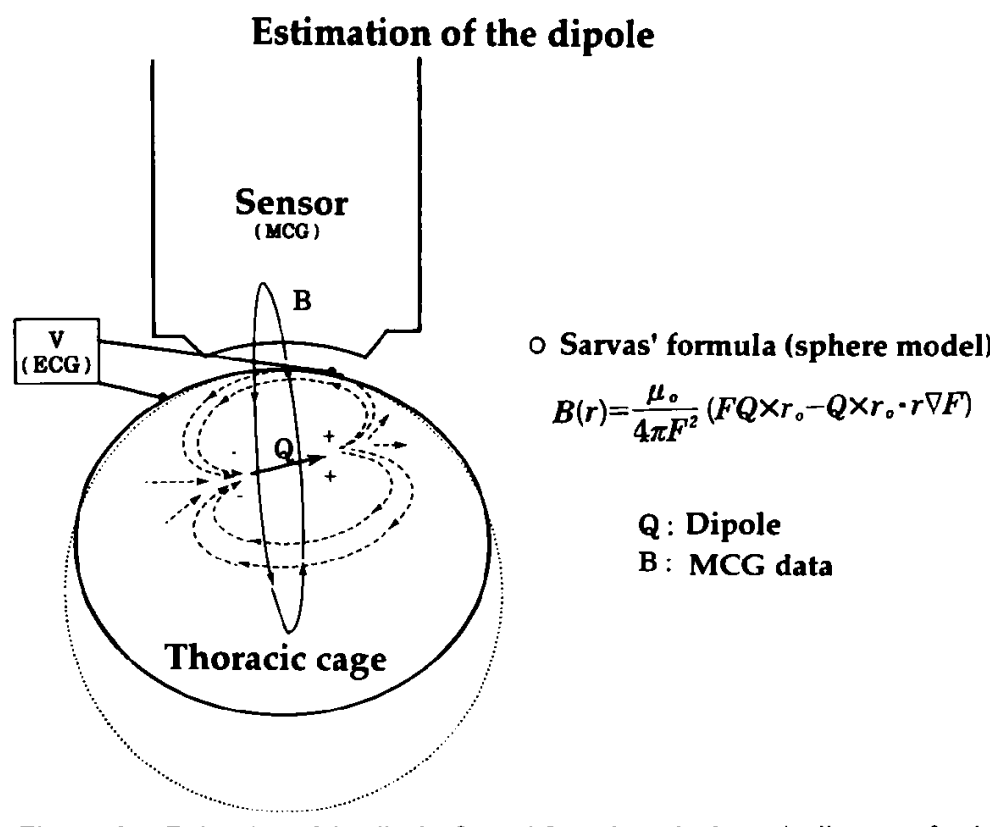

Figure 3. Estimation of the dipole. Sarvas' formula and schematic diagram of estimation of dipole. A single equivalent current dipole $(Q)$ at point $r_{0}$ in the sphere produces magnetic field $B$ at point $r$ outside the sphere. The relationship between $B$ and $Q$ is displayed as Sarvas' formula on the right. The method to calculate inverse solution is described in the text. $F$, a, a, and $F$ were defined as follows. Symbols in italics indicate a vector. $\mathbf{F}=\mathbf{a}\left(\mathrm{r}_{0} \mathbf{a}+\mathrm{r}_{0}{ }^{2}-r \cdot r_{0}\right), \mathbf{a}=|a|, a=r_{0}-r, \nabla \mathbf{F}$ is the gradient of $F$ with respect to $\mathbf{r}_{0}$.

enous conductivity. ${ }^{12)}$ The radius of the thoracic plane was estimated in each subject and recorded by a digitizer. The estimated sphere was positioned and adjusted for a best-fit to the region of the thorax under the biomagnetometer probe during data acquisition according to the digitizer recorded data. The source localization of a single equivalent current dipole (ECD) at each instance of the excitation was then estimated from the distributions of the measured magnetic field using Sarvas' formula for the sphere model (Figure 3). ${ }^{12}$

To solve an inverse problem of the point source, we tentatively assumed electrical dipoles within a sphere and theoretically calculated the magnetic fields outside the sphere. Among the assumed dipoles, in the end we adopted the best fit dipole, which gave the least square error between the measured magnetic field strength and the calculated one, as the estimated ECD. An estimated single ECD was used to approximate the best position of the electrical activity of the heart at each instant. The biomagnetometer SMI-1004 displays the results of an estimated ECD as a 3 dimensional localization of the dipole origin, its magnitude and the direction of the current dipole in each instance with the positions of several pre-determined points. The beginning of ventricular excitation was dc- 
fined as when the magnitude of the slope of ECD increased upwards and exceeded $0.8 \mathrm{pT}$.

Dipolarity analysis: To evaluate the non-dipolarity component of each instance of the excitation, we defined the non-dipole value (NDV) as,

$$
\mathrm{NDV}=\left(\Sigma\left(\mathrm{m}_{\mathrm{i}}-\mathrm{c}_{\mathrm{i}}\right)^{2} / \Sigma \mathrm{m}_{\mathrm{i}}^{2}\right) \times 100(\%),
$$

where $m_{i}$ and $c_{i}$ indicate the magnetic field strength at the channel $i$ of measured values and of theoretical values calculated using the estimated ECD, respectively. ECD was estimated as previously described for the best approximation of the magnetic field distribution at the moment of excitation to minimize the $\left(\mathrm{m}_{\mathbf{i}}-\mathrm{c}_{\mathrm{i}}\right)^{2}$ value. If the value became smaller, it was interpreted as a low non-dipole component and the magnetic field distribution was well explained by a single estimated ECD. If the $\left(\mathrm{m}_{\mathrm{i}}-\mathrm{c}_{\mathrm{i}}\right)^{2}$ value became larger, it was interpreted as an increase in the non-dipole component, that is, the existence of multiple dipoles. The NDV of each timing of excitation from the 222 channels (sensor unit 6 positions times 37 channels each) were calculated and displayed by the SMI-1004 biomagnetometer. We tentatively expressed a NDV over $5 \%$ as a high NDV, which suggests an increase in the non-dipole component at the moment. We excluded high NDV in the initial phase of QRS (within $5 \mathrm{~ms}$ from the onset of QRS) because a relatively low ECD magnitude might be significantly contaminated by the background noise.

\section{Results}

Distribution of isomagnetic fields: Serial isomagnetic field maps were constructed for every $5 \mathrm{~ms}$ of the ventricular excitation for all 16 adult subjects. An isomagnetic field map of one maximum and one minimum with a linear border (zero) line or hyperbolic isomagnetic lines was assumed to be a magnetic field compatible with a single dipole. Isomagnetic maps of case A (Figure 4) show nearly the single dipole pattern up to $30 \mathrm{~ms}$ of excitation. At $40 \mathrm{~ms}$ of excitation, the map shows two minima with round zero or isomagnetic lines, suggesting the presence of multiple dipoles. Isomagnetic maps of case B (Figure 5) show nearly the single dipole pattern at 5, 15,30, and $40 \mathrm{~ms}$ of excitation. However, the maps at $25 \mathrm{~ms}$ and $50 \mathrm{~ms}$ exhibit two maxima or curved zero lines, suggesting the presence of multiple dipoles. Therefore, single dipole or multi dipole patterns appeared during one cycle of ventricular activation.

Serial estimations of non-dipolarity during cardiac excitation: The nondipole values (NDV) of every $1 \mathrm{~ms}$ were calculated from the onset of QRS to 60 $\mathrm{ms}$ in cases $A$ and $B$ (Figures 4 and 5). Case A showed one peak of a high NDV over $5 \%$ after $38 \mathrm{~ms}$ from the onset of the excitation (Figure 6). Case B showed 


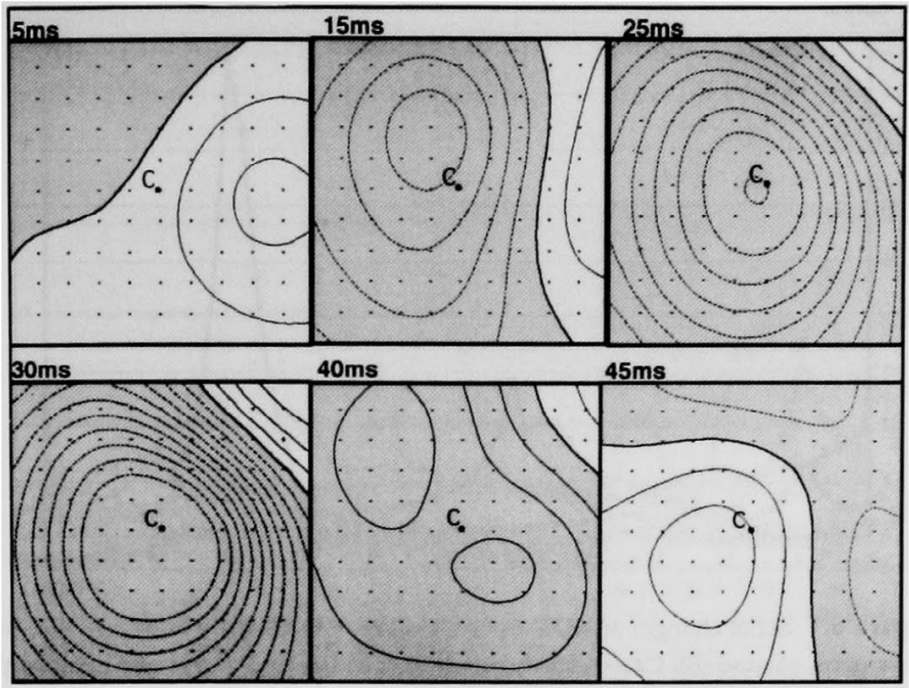

Figure 4. Serial isomagnetic maps of a representative case (A) from the beginning of QRS. White areas are positive and shaded areas are negative. A single dipole pattern of the isomagnetic maps is seen from $5 \mathrm{~ms}$ to $30 \mathrm{~ms}$. At $40 \mathrm{~ms}$ and $45 \mathrm{~ms}$, two minima with curved zero lines are seen, indicating the presence of a multiple dipole. $\mathrm{C}$; point C of Figure 1.

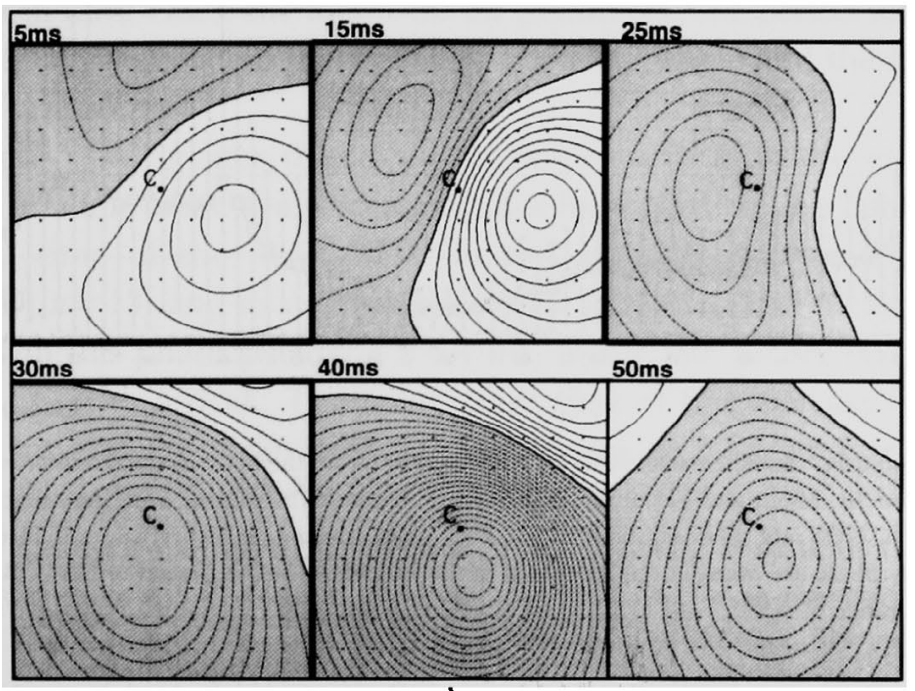

Figure 5. Serial isomagnetic maps of another representative case (B) from the beginning of QRS. White areas are positive and shaded areas are negative. The zero line is not curved in the map from $15 \mathrm{~ms}$ to $30 \mathrm{~ms}$. At $50 \mathrm{~ms}$, there are two maxima, suggesting the presence of a multiple dipole. $\mathrm{C}$ : point $\mathrm{C}$ of Figure 1. 


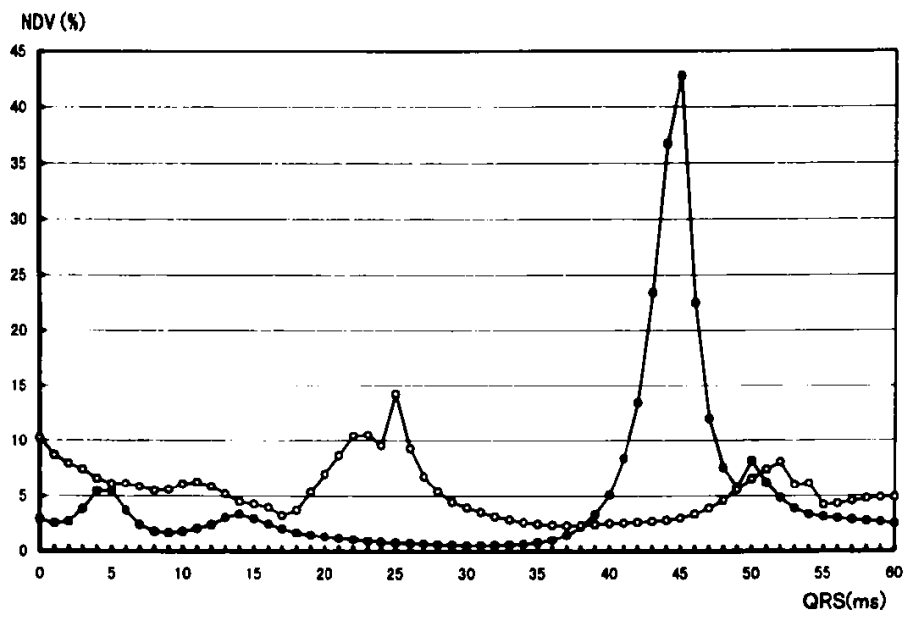

Figure 6. Serial changes of NDV from the onset of QRS to $60 \mathrm{~ms}$ in two representative cases, (A) and (B). Case A (closed circle) is A shown in Figure 4 and case B (open circle) is B in Figure 5. Case A showed one NDV peak at around $40 \mathrm{~ms}$ from the beginning of QRS and case $B$ showed two peaks (25 ms and around $50 \mathrm{~ms}$ ). These non-dipolarity characteristics correspond well with the findings in isomagnetic maps of Figures 4 and 5 .

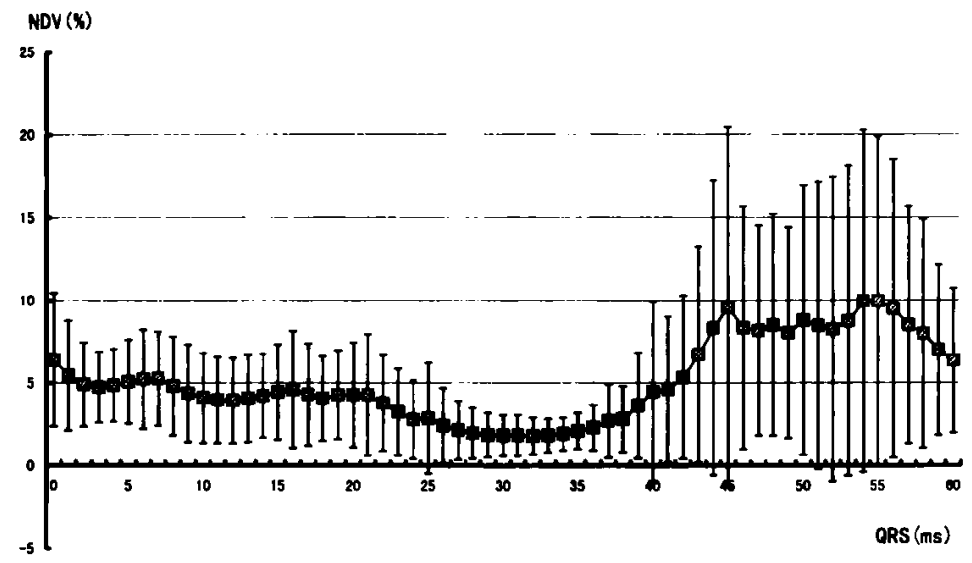

Figure 7. Serial changes in mean NDV and SD from the onset of QRS to $60 \mathrm{~ms}$ in 16 adults. Mean and SD of NDV showed minimum at $32-34 \mathrm{~ms}$ from the onset of QRS. NDV are relatively high in the early phase and much higher values than the early phase are noted in the late phase of the excitation. Square: mean of NDV. Bar: $\mathrm{SD}$ range of $\mathrm{NDV}$.

two peaks of high NDVs, in the early phase at $25 \mathrm{~ms}(12.8 \%)$ and the late phase at $48 \mathrm{~ms}$ (over $5 \%$ ). The other 14 adult cases were also evaluated for NDV from the beginning to $60 \mathrm{~ms}$ of QRS at $1 \mathrm{~ms}$ intervals. The combined results for the 16 adult cases including the two shown in Figure 6, are presented in Figure 7. Mean 


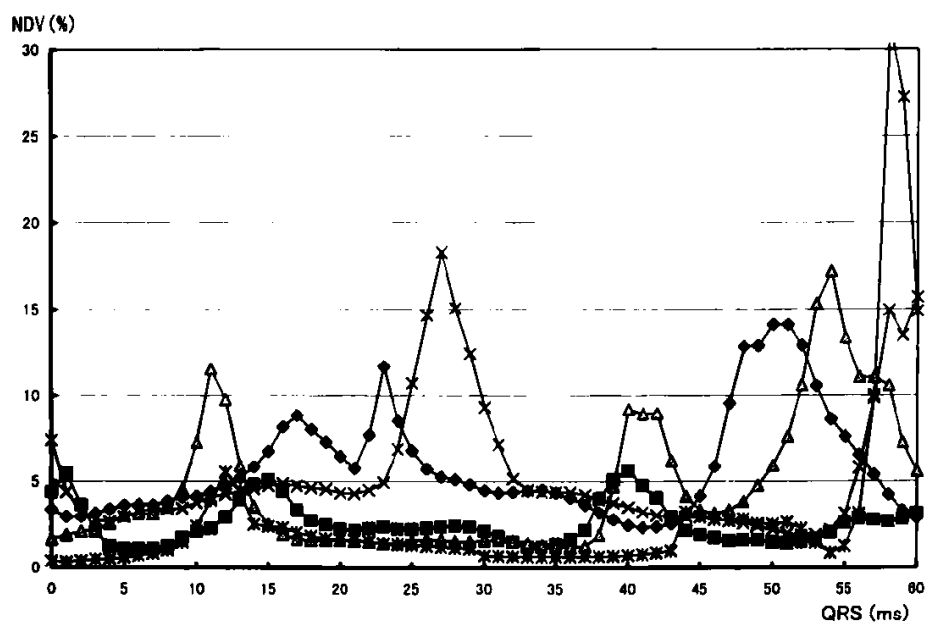

Figure 8. Serial changes in NDV' from the onset of QRS to $60 \mathrm{~ms}$ in all children. The children also exhibited low mean NDV and SD at around $35 \mathrm{~ms}$ from the onset of QRS. All cases had two .NDY peaks and the values are somewhat higher than those of adults.

NDV and its deviation exhibited the lowest value between 30 and $35 \mathrm{~ms}$ of QRS onset. Outside of this period, the NDV were close to or above the level of $5 \%$. If we exclude the NDV from the evaluation at the initial $5 \mathrm{~ms}$ of QRS because the absolute values of the magnetic field were small, high NDV were present in the two phases in most cases: the first $6-20 \mathrm{~ms}$ period and the period after about 40 ms of QRS. We designated these periods the early phase and the late phase of high NDV, respectively.

A high NDV greater than $5 \%$ was observed in both the early and late phases in 13 adult cases. Only 3 cases had a high NDV in the late phase only. The mean time of the peak NDV in the early phase was $14.5 \pm 6.5 \mathrm{~ms}$ (mean $\pm \mathrm{SD}, n=13$ ) and its mean value was $9.3 \pm 3.0 \%$. The mean duration of high NDV in this phase lasted $7.4 \pm 4.4 \mathrm{~ms}$. In the late phase, NDV increased over than $5 \%$ with a peak. The mean onset time of this increase in NDV was $43.6 \pm 5.0 \mathrm{~ms}$ and the mean peak time and value of NDV in late phase were $49.6 \pm 6.5 \mathrm{~ms}$ and $21.5 \pm 10.5 \%(n=16)$, respectively.

All 5 children exhibit a high NDV greater than $5 \%$ in both the early and late phases (Figure 8). The mean time of the peak NDV at the early phase was $17.6 \pm 7.1 \mathrm{~ms}$ (mean $\pm \mathrm{SD}, n=5$ ) and the mean value was $10.5 \pm 5.4 \%$. The mean duration of high NDV at this phase lasted $6.2 \pm 6.4 \mathrm{~ms}$. In the late phase, NDV increased over than $5 \%$ with a peak. The mean onset time of this increase in NDV was $47.8 \pm 8.8 \mathrm{~ms}$ and the mean peak time and value of NDV in late phase were $52.6 \pm 7.9 \mathrm{~ms}$ and $16.8 \pm 7.9 \%(n=5)$, respectively. 


\section{Discussion}

The present study examined the dipolarity characteristics of normal excitation of the heart using magnetic field measurements. The results indicate that at 32-34 ms from the beginning of QRS, the mean NDV and its deviation were minimum, suggesting a small non-dipole component at this moment of the ventricular activation. Second, all subjects, including the children, exhibited a high NDV after 35 ms of excitation. Third, in most of the adults and all the children, there was another, although less marked, peak of NDV in the early phase at 6$25 \mathrm{~ms}$ of QRS. These results indicate that non-dipole components of ventricular excitation were present in the two phases, the early and late phases of QRS, in most of the normal subjects.

Durrer et al. ${ }^{13)}$ examined activation patterns in isolated human hearts, finding that left ventricular excitation began from 3 areas and rapidly increased in size during the next 5 to $10 \mathrm{~ms}$. In the present study, a portion of this initial activation may not be detected as an increase in NDV in the initial $5 \mathrm{~ms}$ because this $5 \mathrm{~ms}$ period was excluded from the analysis. The left ventricular activation became confluent 15 to $20 \mathrm{~ms}$ after the onset of excitation. In the right ventricle, the activation started near the insertion of the anterior papillary muscle about 5 to $10 \mathrm{~ms}$ after left ventricular activation. The activation then progressed, leading to epicardial breakthrough after about $20 \mathrm{~ms}$ of QRS. ${ }^{13)}$ The increase in NDV in the early phase observed in our study appeared to reflect these propagation patterns of the right ventricle simultaneously with that in the left ventricle. Relative increases in the areas of right ventricular activation resulted in the appearance of multiple excitation waves, reflecting an increase in the non-dipole component of cardiac excitation. This explanation may agree partly with the timing of right ventricular breakthrough (mean $17 \mathrm{~ms}$ ) by the epicardial map reported by Wyndham et al. ${ }^{14)}$ Furthermore, the 5 children showed very similar patterns for NDV distribution throughout cardiac excitation, including the appearance of two peaks. High NDV in the early phase may also support the involvement of right ventricular excitation in increasing NDV since the contribution of right compared to left ventricular excitation is more dominant in children than in adults.

Durrer et al. ${ }^{13)}$ also demonstrated that the left ventricular activation front almost completely spread throughout the endocardial layer $30 \mathrm{~ms}$ from the onset of excitation and the activation continued to progress in a perpendicular direction towards the epicardial surface. Thirty ms after the onset of excitation, the activation in the left ventricle was directed toward the epicardial surface. The activation in the interventricular septum from the left to the right ventricle was completed in this phase of the excitation. Because of the dominant left ventricular 
mass, total activation at this moment may be represented by the main left ventricular excitation around 30-35 ms from the QRS onset. An increase in NDV in the late phase may be a reflection produced by the multiple activation fronts toward the posterobasal or posterolateral areas of the left ventricle, and the pulmonary conus area in the right ventricle. In addition, the small magnitude of the magnetically measured ECD in this phase may contribute to increased NDV due to the contamination by background noise.

The dipolar characteristics of the heart in situ have been previously reported by Taccardi $\left.{ }^{5}\right)$ and Tsunakawa et ${ }^{(6)}$ using electrical measurements. Taccardi showed that the thoracic distribution of the cardiac activity was a single dipole during a majority of QRS in normal subjects. He also showed that non-dipole components, represented by multi maxima or minima in isopotential distributions of body surface maps, were seen at 20-35 ms and in the last third of QRS. Tsunakawa et al defined the term residue as an index of the non-dipole component corresponding to NDV in our study. NDV, as described previously, was calculated from the least square error value and the residue was defined as its square root value. Therefore, NDV (around $5 \%$ ) in the present study were comparable to the residue values (around $20 \%$ ). They also reported that there were two types of distribution patterns for the residue during QRS. Eleven of their 27 subjects showed two residue peaks at around $24.4 \mathrm{~ms}$ (mean) and $48.6 \mathrm{~ms}$ (mean) from the onset of QRS. These results agree well with our NDV results, which showed two peaks at $14.1 \mathrm{~ms}$ (mean) and $43.6 \mathrm{~ms}$ (mean) from the onset of QRS in the adults. Only one peak residue in the late phase (mean $46.6 \mathrm{~ms}$ ) of excitation was observed in 14 subjects in their study. Although the relative incidences of the two types are somewhat different in the two studies, the results confirm the existence of two peaks of a non-dipolar component in normal excitation of the heart.

The present study demonstrated serial and quantitative non-dipole characteristics of the heart potential in situ in normal adults and children by magnetic measurements. A single dipole estimation by MCG showed a low NDV relative to residue of electrical measurements. At the late phase of the excitation, a higher NDV with a longer duration than that of the early phase of the excitation was observed. The result suggests that a single dipole estimation by MCG may be applied to clinical evaluation and may be a potentially useful in evaluating the pathological conditions of cardiac excitation.

\section{ACKNOWLEDGMENTS}

The authors wish to thank Mr Koichi Nitta and Ms Kanako Naito of Sumitomo Metal Industries Ltd. for their skillful technical assistance. 


\section{References}

1. Kamakura S, Shimomura K, Ohe T, Matsuhisa M, Toyoshima $H$. The role of initial minimum potentials on body surface maps in predicting the site of accessory pathways in patients with WolffParkinson-White syndrome. Circulation 1986; 74: 89-96.

2. Moshage W, Achenbach S, Göhl K, et al. Biomagnetic localization of cardiac arrhythmias. Radiology 1991; 180: 685-92.

3. Moshage W', Achenbach S, Gohl K, Bachmann $\mathrm{K}$. Evaluation of the non-invasive localization accuracy of cardiac arrhythmias attainable by multichannel magnetocardiography (MCG). Int J Cardiac Imaging 1996; 12: 47-59.

t. Scher $\mathrm{AM}$, Young $\mathrm{AC} ;$, Meredith WD. Factor analysis of the electrocardiogram: test of electrocardiographic theory. normal heart. Circ Res 1960; 8: 519-26.

5. Taccardi B. Distribution of heart potentials on the thoracic surface of the normal human subject. Circ Res 1963; 12: 341-52.

6. Tsunakawa $\mathrm{H}$, Hoshino $\mathrm{K}$, Kanesaka $\mathrm{S}$, et al. Dipolarity and dipole location during $\mathrm{QRS}$ and $\mathrm{T}$ waves in normal men estimated from body surface potential distribution. Jpn Heart J 1985; 26: 319-34.

7. Baule GN, McFee R. Detection of the magnetic field of the heart. Am Heart J 1963; 66: 95-6.

8. Schneider $\mathrm{S}$. Hoenig E, Reichenberger $\mathrm{H}$. et al. Multichannel biomagnetic system for study of electrical activity in the brain and heart. Radiology 1990; 176: 825-30.

9. Hart G. Biomagnetometry: imaging the heart's magnetic field. Br Heart J 1991; 65: 61-2.

10. Nakaya Y, Mori H. Magnetocardiography. Clin Phys Physiol Mcas 1992; 13: 191-229.

11. Hren R, Zhang X, Stroink G. Comparison between electrocardiographic and magnetocardiographic inverse solutions using the boundary element method. Med Biol Eng Comput 1996; 34: 110-4.

12. Sarvas J. Basic mathematical and electromagnetic concepts of the biomagnetic inverse problem. Phys Med Biol 1987; 32: 11-22.

13. Durrer D, Van Dam RT, Freud GE, Junse MJ, Mijler FL, Arzbaecher RC. Total excitation of the isolated human heart. Circulation 1970; 41: 899-912.

14. Wyndham CR, Meeran MK, Smith T, et al. Epicardial activation of the intact human heart without conduction defect. Circulation 1979; 59: 161-8. 